\title{
Optimized Schwarz Methods and model adaptivity in electrocardiology simulations
}

\author{
Luca Gerardo-Giorda, Lucia Mirabella, Mauro Perego and Alessandro Veneziani
}

\begin{abstract}
The Bidomain model is nowadays one of the most accurate mathematical descriptions of the action potential propagation in the heart. However, its numerical approximation is in general fairly expensive as a consequence of the mathematical features of this system, and several works have been devoted to devise effective solvers and preconditioners, $[10,11,15]$ among others. A simplification of this model, called Monodomain problem is often adopted in order to reduce computational costs of the numerical solution of the cardiac potential. The latter model is however less accurate. A possible trade-off between accuracy and cost is a model adaptive strategy. The computational domain is subdivided into regions, coupled through an Optimized Schwarz Method, in which either the Bidomain or the Monodomain problem is solved, according to an a-posteriori model error estimator following the spatio-temporal evolution of the action potential propagation. Here we present a possible implementation of this approach, following up previous works on the error estimation and the Optimized Schwarz coupling.
\end{abstract}

\section{The Numerical Model}

At the macroscopic level, the myocardial tissue is regarded as the superposition of two continuous and anisotropic media, the intra-cellular and the extra-cellular one.

Luca Gerardo-Giorda

BCAM - Basque Center for Applied Mathematics, Bilbao, Spain, e-mail: lgerardo@ bcamath.org

Lucia Mirabella

"W.H. Coulter" Dept. Biomed. Engrg., GaTech, Atlanta, GA, USA e-mail: lucia.mirabella@bme.gatech.edu

Mauro Perego

Dept. of Sci. Comp., Florida State University, Thallahassee, FL, USA e-mail: mperego@fsu.edu

Alessandro Veneziani

Dept. of Math. and CS, Emory University, Atlanta, GA, USA e-mail: ale@mathcs.emory.edu 
They coexist at each point and are connected by a cell membrane. The tissue conductivity depends upon its cells orientation, and in the most general case the associated tensor is anisotropic [7, 14]. In any point $x \in \Omega$, where $\Omega$ is the spatial domain under consideration, it is possible to identify an orthonormal triplet of directions, $a_{l}(x), a_{t}(x), a_{n}(x)$, with $a_{l}(x)$ parallel to the fibers direction, and we denote by $\sigma_{i, e}^{l}$, $\sigma_{i, e}^{t}$, and $\sigma_{i, e}^{n}$ the corresponding intra and extracellular conductivity coefficients. The conductivity tensors are given by

$$
\mathbf{D}_{\tau}(x)=\sigma_{\tau}^{l}(x) a_{l}(x) a_{l}^{T}(x)+\sigma_{\tau}^{t}(x) a_{t}(x) a_{t}^{T}(x)+\sigma_{\tau}^{n}(x) a_{n}(x) a_{n}^{T}(x), \quad \tau=i, e .
$$

We assume that $\mathbf{D}_{i, e}$ fulfill in $\Omega$ a uniform elliptic condition.

The Bidomain model. The Bidomain model is a nonlinear reaction-diffusion system of parabolic type describing the spatio-temporal dynamics of the intra and extracellular potentials, denoted by $u_{i}$ and $u_{e}$, while the cell membrane is regarded as dislocated in the domain [2]. We rely in this paper on a non-symmetric formulation in terms of the transmembrane potential $u=u_{i}-u_{e}$, and the extracellular one [4]. We denote by $\mathbf{u}=\left(u, u_{e}\right)^{T}$ the unknown, and by letting

$$
\mathbf{D}=\left[\begin{array}{cc}
\frac{\sigma_{e}^{l} \mathbf{D}_{i}}{\sigma_{i}^{l}+\sigma_{e}^{l}} & \frac{\sigma_{e}^{l} \mathbf{D}_{i}-\sigma_{i}^{l} \mathbf{D}_{e}}{\sigma_{i}^{l}+\sigma_{e}^{l}} \\
\mathbf{D}_{i} & \mathbf{D}_{i}+\mathbf{D}_{e}
\end{array}\right] \quad \mathbf{E}_{1}=\left[\begin{array}{ll}
1 & 0 \\
0 & 0
\end{array}\right] \quad \mathbf{e}_{1}=\left[\begin{array}{l}
1 \\
0
\end{array}\right]
$$

the Bidomain system reads

$$
\chi C_{m} \mathbf{E}_{1} \frac{\partial \mathbf{u}}{\partial t}-\nabla \cdot \mathbf{D} \nabla \mathbf{u}+\chi I_{i o n}(u) \mathbf{e}_{1}=\mathbf{I}^{a p p}
$$

where $I_{i o n}(u)$ is a nonlinear function of the transmembrane potential $u$, specified by a ionic model, and where $\mathbf{I}^{a p p}$ represent the applied current stimuli. Several ionic models are available in literature, from more phenomenological to more accurate ones, but the choice of the nonlinear term $I_{i o n}(u)$ does not have any influence on the procedure highlighted in what follows. The problem is completed by suitable initial conditions, and by homogeneous Neumann boundary conditions on $\partial \Omega$, modeling an insulated myocardium. The transmembrane potential $u$ is then uniquely determined from (2), while the extracellular potential $u_{e}$ is determined up to a function of time, whose value is usually obtained by imposing that $\int_{\Omega} u_{e}=0$.

The Monodomain model. The Monodomain model is a simplified model for the propagation of the electrical stimulus, based upon a proportionality assumption between the intracellular and the extracellular conductivity tensors, namely assuming $\mathbf{D}_{e}=\lambda \mathbf{D}_{i}$, where $\lambda$ is a constant to be properly chosen. We assume here $\lambda=\sigma_{e}^{l} / \sigma_{i}^{l}$ [6], and the Monodomain model reads

$$
\chi C_{m} \frac{\partial u}{\partial t}-\nabla \cdot \frac{\sigma_{e}^{l} \mathbf{D}_{i}}{\sigma_{i}^{l}+\sigma_{e}^{l}} \nabla u+\chi I_{i o n}(u)=I^{a p p},
$$


still coupled with suitable initial conditions, and homogeneous Neumann boundary conditions on $\partial \Omega$. Differently from the Bidomain, the Monodomain model features a unique solution and is way cheaper to solve numerically. In absence of applied currents, the Monodomain model is accurate enough to catch the desired dynamics and effects of the action potential propagation [12]. However, the Bidomain model becomes necessary when current stimuli are applied in the extracellular space. Also, the Monodomain is inadequate to simulate defibrillation [16].

\subsection{Numerical approximation}

Time integration. For simplicity in presentation, we consider a fixed time step $\Delta t$, and we denote with superscript $n$ the variables computed at time $t^{n}=n \Delta t$. Both the Bidomain (2) and the Monodomain equations (3) are advanced in time by a semi-implicit scheme, where the nonlinear term (the ionic current) is evaluated at the previous time steps. More precisely, moving from $t^{n}$ to $t^{n+1}$ we solve in $\Omega$

$$
\chi C_{m} \mathbf{E}_{1} \frac{\mathbf{u}^{n+1}-\mathbf{u}^{n}}{\Delta t}-\nabla \cdot \mathbf{D} \nabla \mathbf{u}^{n+1}=\mathbf{I}^{a p p}-\chi I_{\text {ion }}\left(u^{n}\right) \mathbf{e}_{1}
$$

for the Bidomain system, and

$$
\chi C_{m} \frac{u^{n+1}-u^{n}}{\Delta t}-\nabla \cdot \frac{\sigma_{e}^{l} \mathbf{D}_{i}}{\sigma_{i}^{l}+\sigma_{e}^{l}} \nabla u^{n+1}=\mathbf{I}^{a p p}-\chi I_{\text {ion }}\left(u^{n}\right)
$$

for the Monodomain one.

Space discretization. Both Bidomain (4) and Monodomain (5) models are discretized in space by finite elements. When solving the Bidomain system, the unknowns of the fully discrete problem are represented by the vector $\left(u_{h}, u_{e, h}\right)^{T}$, storing the nodal values of the transmembrane and extracellular potentials. The matrix associated with the discrete Bidomain models is given by

$$
\mathbf{B}=\left[\begin{array}{ll}
\mathbf{B}_{u u} & \mathbf{B}_{u e} \\
\mathbf{B}_{e u} & \mathbf{B}_{e e}
\end{array}\right]=\left[\begin{array}{ll}
\frac{\chi C_{m}}{\Delta t} \mathbf{M}+\frac{\sigma_{e}^{l}}{\sigma_{i}^{l}+\sigma_{e}^{l}} \mathbf{K}_{i} & \frac{\sigma_{e}^{l}}{\sigma_{i}^{l}+\sigma_{e}^{l}} \mathbf{K}_{i}-\frac{\sigma_{i}^{l}}{\sigma_{i}^{l}+\sigma_{e}^{l}} \mathbf{K}_{e} \\
\mathbf{K}_{i} & \mathbf{K}_{i}+\mathbf{K}_{e}
\end{array}\right],
$$

where $\mathbf{M}$ is the mass matrix and $\mathbf{K}_{i, e}$ are the stiffness matrices associated with the chosen finite elements space.

When solving the Monodomain system, the unknown of the fully discrete problem is $u_{h}$, and the associated matrix is simply block $\mathbf{B}_{u u}$ of the Bidomain matrix. 


\section{A model adaptive strategy}

Stemming from the observation that the Monodomain error is concentrated around the wavefront (particularly crosswind with respect to the fibers, see Figure 1), we consider here a model adaptive strategy that solves the Bidomain only where actually needed. In a first implementation of this approach [9] an intermediate $\mathrm{Hy}$ bridomain model based on a suitable a posteriori model estimator was introduced. The Hybridomain assembles the block $\mathbf{B}_{u e}$ only in correspondence with the nodes identified as Bidomain ones by the model estimator, while the second equation stays untouched. This simplifies significantly the implementation of the adaptive procedure, however the computational advantage is limited, since also in the Monodomain regions an extended problem with the same size of the Bidomain one is solved. An alternative procedure consists of a genuine heterogenous coupling by splitting the domain. This coupling guarantees a significant reduction of the computational costs, but raises non trivial issues when matching the two models, featuring a different size. This has been considered in [6], where the Optimized Schwarz method has been advocated for the heterogeneous coupling.

From the practical standpoint, an immediate idea for setting up a model adaptive solver would be to use the a posteriori error estimator for detecting the regions where to solve the Bidomain problem and then to couple these subdomains with the Monodomain regions, so to have the Bidomain model capturing the wavefron propagation. However, this approach is barely doable. As a matter of fact. the Robintype interface conditions in the Optimized Schwarz setting require the assembly of mass matrices on the interfaces. As a consequence, every time the Bidomain region changes, one should identify the new interfaces and then recompute the matrices, with an additional computational cost that is anticipated to reduce the advantage of the Optimized Schwarz coupling. The model adaptive strategy we propose here relies instead on a a priori subdivision of $\Omega$ into smaller subdomains $\Omega_{j}$. The model error estimator will associate runtime each subdomain with either the Bidomain or the Monodomain problem. In this way, the interfaces matrices needed for the coupling can be computed once at the beginning of the time loop. The non-symmetric formulation of the Bidomain system ensures that by assembling the blocks of every subdomain matrix, also the Monodomain ones are available. Details on the Optimized Schwarz coupling are given in the next Section.


Fig. 1 Error in the propagation of the membrane potential $u$ between Bidomain and Monodomain simulation (from [6]): fibers oriented along the $x$ axis. 


\subsection{Coupling conditions and Optimized Schwarz Methods}

We outline here the coupling conditions for the three different types of interfaces. If the subdomains involved have the same characteristic (Bido/Bido and Mono/Mono) the corresponding solutions are labeled by subscript 1 and 2, while if the subdomains have different characteristics (Bido/Mono) the corresponding solutions are labeled with subscript $B$ and $M$.

Bidomain/Bidomain interface. The coupling conditions on the Bidomain/Bidomain interface have been introduced in [5], and are given by

$$
\begin{aligned}
& \mathbf{n}_{1}^{T} \mathbf{D} \nabla \mathbf{u}_{1}+\alpha_{1} \Sigma \mathbf{u}_{1}=\mathbf{n}_{1}^{T} \mathbf{D} \nabla \mathbf{u}_{2}+\alpha_{1} \Sigma \mathbf{u}_{2} \\
& \mathbf{n}_{2}^{T} \mathbf{D} \nabla \mathbf{u}_{2}+\alpha_{2} \Sigma \mathbf{u}_{2}=\mathbf{n}_{2}^{T} \mathbf{D} \nabla \mathbf{u}_{1}+\alpha_{2} \Sigma \mathbf{u}_{1}, \quad \text { where } \quad \Sigma=\left[\begin{array}{cc}
\frac{\sigma_{e}^{l}}{\sigma_{i}^{l}+\sigma_{e}^{l}} & 0 \\
1 & \frac{\sigma_{i}^{l}+\sigma_{e}^{l}}{\sigma_{i}^{l}}
\end{array}\right] .
\end{aligned}
$$

The convergence of the Optimized Schwarz Algorithm based on the interface conditions (7) was analyzed in [5], where also optimal parameters have been identified by means of Fourier analysis.

Bidomain/Monodomain interface. Due to a dimensional mismatch between the two models, two interface conditions are needed on the Bidomain side of the interface, and one on the Monodomain side [6]. Possible coupling conditions are

$$
\begin{aligned}
& \mathbf{n}_{B}^{T} \frac{\sigma_{e}^{l} \mathbf{D}_{i}}{\sigma_{i}^{l}+\sigma_{e}^{l}}\left(\nabla u_{B}+\nabla u_{e, B}\right)-\mathbf{n}_{B}^{T} \frac{\sigma_{i}^{l} \mathbf{D}_{e}}{\sigma_{i}^{l}+\sigma_{e}^{l}} \nabla u_{e, B}+\frac{\sigma_{e}^{l} \alpha}{\sigma_{i}^{l}+\sigma_{e}^{l}} u_{B}=\mathbf{n}_{B}^{T} \frac{\sigma_{e}^{l} \mathbf{D}_{i}}{\sigma_{i}^{l}+\sigma_{e}^{l}} \nabla u_{M}+\frac{\sigma_{e}^{l} \alpha}{\sigma_{i}^{l}+\sigma_{e}^{l}} u_{B} \\
& \mathbf{n}_{B}^{T} \mathbf{D}_{i}\left(\nabla u_{B}+\nabla u_{e, B}\right)+\mathbf{n}_{B}^{T} \mathbf{D}_{e} \nabla u_{e, B}+\alpha u_{B}+\frac{\sigma_{i}^{l}+\sigma_{e}^{l}}{\sigma_{i}^{l}} \alpha u_{e, B}=\alpha u^{r e s t}
\end{aligned}
$$

for the Bidomain subproblem, and

$$
\mathbf{n}_{M}^{T} \frac{\sigma_{e}^{l} \mathbf{D}_{i}}{\sigma_{i}^{l}+\sigma_{e}^{l}} \nabla u_{M}+\frac{\sigma_{e}^{l} \alpha}{\sigma_{i}^{l}+\sigma_{e}^{l}} u_{M}=\mathbf{n}_{M}^{T} \frac{\sigma_{e}^{l} \mathbf{D}_{i}}{\sigma_{i}^{l}+\sigma_{e}^{l}}\left(\nabla u_{B}+\nabla u_{e, B}\right)-\mathbf{n}_{M}^{T} \frac{\sigma_{i}^{l} \mathbf{D}_{e}}{\sigma_{i}^{l}+\sigma_{e}^{l}} \nabla u_{e, B}+\frac{\sigma_{e}^{l} \alpha}{\sigma_{i}^{l}+\sigma_{e}^{l}} u_{B}
$$

for the Monodomain one. To cope with the mismatch, the second condition in (8) is a transparent boundary condition, designed to avoid spurious reflexions off the interface for the extracellular potential wave. The convergence of the Optimized Schwarz Algorithm based on the interface conditions (8)-(9) was analyzed in [6], where also optimal parameters has been identified by means of Fourier analysis.

Monodomain/Monodomain interface. The Optimized Schwarz coupling is way simpler on the interface between two Monodomain regions. The Monodomain model is a parabolic equation equipped with a nonlinear reaction term. The IMEX temporal integration scheme reduces it, within the single time step, to the solution of 
a linear steady reaction-diffusion problem, whose solution by means of Optimized Schwarz Methods has been widely studied, and an optimal parameter has been identified [3]. The coupling on the interface is thus given by

$$
\begin{aligned}
& \mathbf{n}_{1}^{T} \frac{\sigma_{e}^{l} \mathbf{D}_{i}}{\sigma_{i}^{l}+\sigma_{e}^{l}} \nabla u_{1}+\alpha^{o p t} u_{1}=\mathbf{n}_{1}^{T} \frac{\sigma_{e}^{l} \mathbf{D}_{i}}{\sigma_{i}^{l}+\sigma_{e}^{l}} \nabla u_{2}^{p}+\alpha^{o p t} u_{2}^{p} \\
& \mathbf{n}_{2}^{T} \frac{\sigma_{e}^{l} \mathbf{D}_{i}}{\sigma_{i}^{l}+\sigma_{e}^{l}} \nabla u_{2}+\alpha^{o p t} u_{2}=\mathbf{n}_{2}^{T} \frac{\sigma_{e}^{l} \mathbf{D}_{i}}{\sigma_{i}^{l}+\sigma_{e}^{l}} \nabla u_{1}+\alpha^{o p t} u_{1}
\end{aligned}
$$

\subsection{The model error estimator}

For choosing between a Bidomain or Monodomain simulation within the single subdomain we need a reliable model error estimator. An efficient a posteriori error estimator was introduced in [9], based on the extracellular potential computed from an extended Monodomain solution. To further reduce the computational cost, we compute only a Monodomain solution $u_{M}$ over the whole domain. We let $\mathbf{D}_{e}=\frac{\sigma_{e}^{l}}{\sigma_{i}^{l}} \mathbf{D}_{i}+\mathbf{D}_{\varepsilon}$, and the model estimator is computed at the subdomain level
as

$$
\zeta_{j}^{2}=\int_{t^{n}}^{t^{n+1}} \int_{\Omega_{j}} \nabla u_{M} \frac{\sigma_{i}^{l} \mathbf{D}_{\varepsilon}}{\sigma_{i}^{l}+\sigma_{e}^{l}}\left(\mathbf{D}_{i}^{-1}+\mathbf{D}_{e}^{-1}\right) \frac{\sigma_{i}^{l} \mathbf{D}_{\varepsilon}}{\sigma_{i}^{l}+\sigma_{e}^{l}} \nabla u_{M} d x d t
$$

The value $\zeta_{j}^{2}$ is an upper bound for the error between the Monodomain and the Bidomain model within the $\Omega_{j}$ [9]. The Bidomain model is then activated in $\Omega_{j}$ whenever $\zeta_{j}^{2}$ exceeds a given threshold $\tau_{j}$, depending on the size of the subdomain.

\subsection{The model adaptive algorithm}

We summarize here the resulting model adaptive strategy, by highlighting the preprocessing and the runtime phases.

\section{Preprocessing}

1. Split the computational domain into non-overlapping subregions $\Omega_{j}(j=1, \ldots, N)$.

2. Identify the interfaces $\Gamma_{i j}$ between subdomains $\Omega_{i}$ and $\Omega_{j}$.

3. Assemble the local matrices $\mathbf{B}_{u u}^{j}, \mathbf{B}_{u e}^{j}, \mathbf{B}_{e u}^{j}, \mathbf{B}_{e e}^{j}$

4. Assemble the interface mass matrices $\mathbf{M}_{\Gamma_{i j}}$

5. Compute the incomplete ILU factorization of the local $\mathbf{B}_{u u}^{j}$ and $\mathbf{B}_{e e}^{j}$ matrices

Runtime (time step $t^{n} \rightarrow t^{n+1}$ )

1. Run a Monodomain simulation at time $t^{n+1}$ over the whole domain $\Omega$

2. Evaluate the a-posteriori model estimator and compute the local indicator 
3. For all subdomains where the local indicator is above a given threshold, activate Bidomain

4. Run a few iteration of the Optimized Schwarz Algorithm using the solution computed in Step 1. as initial guess

5. Advance to time $t^{n+1}$

A dynamical allocation of tasks is under investigation to properly balance, in real problems, the load of each processor in the parallel solver.

\section{Preliminary numerical results}

Numerical results in this section have the purpose to show the effectiveness of the model adaptive method: for this reason we consider here only 2D simulations. The numerical tests are run in Matlab ${ }^{\circledR}$ 7.5. The Bidomain problems are solved with by a flexible GMRES (f-GMRES) right preconditioned by the Block-triangular preconditioner introduced in [4], while the Monodomain problems are solved by a CG preconditioned by an ILU factorization.

We consider the strip $\Omega=[0,3] \times[0,1]$ subdivided into the three noverlapping subdomains $\Omega_{1}=[0,1] \times[0,1], \Omega_{2}=[1,2] \times[0,1]$, and $\Omega_{3}=[2,3] \times[0,1]$. The fibers are oriented with the principal direction perpendicular to the interfaces, and we impose a stimulus on the whole left boundary of $\Omega_{1}$. The ionic model is the RogersMcCulloch one [13]. We plot in Figure 2 the wavefront position at different times (top row), and the activated subdomains (bottom row).

A more detailed presentation of the method will be the subject of a forthcoming work. Further work needs to be done to identify the proper trade-off between the number of subdomains, and the size of the Bidomain region surrounding the wavefront, and to properly handle the processors load balance in a parallel architecture.

\section{References}

1. R. H. Clayton, O. M. Bernus, E. M. Cherry, H. Dierckx, F. H. Fenton, L. Mirabella, A. V. Panfilov, F. B. Sachse, G. Seemann, H. Zhang, Models of cardiac tissue electrophysiology: Progress, challenges and open questions, Progress in biophysics and molecular biology 104, pp. 22-48, 2011.

2. P. Colli Franzone, L. Pavarino, G. Savaré. Computational electrocardiology: mathematical and numerical modeling, in Complex Systems in Biomedicine - A. Quarteroni, L. Formaggia, and A. Veneziani, editors. Springer, Milan, 2006.

3. M.J. Gander. Optimized Schwarz methods. SIAM J. Num. Anal., 44(2), pp. 699-731, 2006.

4. L. Gerardo-Giorda, L. Mirabella, F. Nobile, M. Perego, and A. Veneziani. A model-based block-triangular preconditioner for the Bidomain system in electrocardiology. J. Comp. Phys., 228, pp. 3625-3639, 2009.

5. L. Gerardo-Giorda and M. Perego, Optimized Schwarz Methods for the Bidomain system in electrocardiology M2AN, September 2012, DOI: http://dx.doi.org/10.1051/m2an/2012040. 

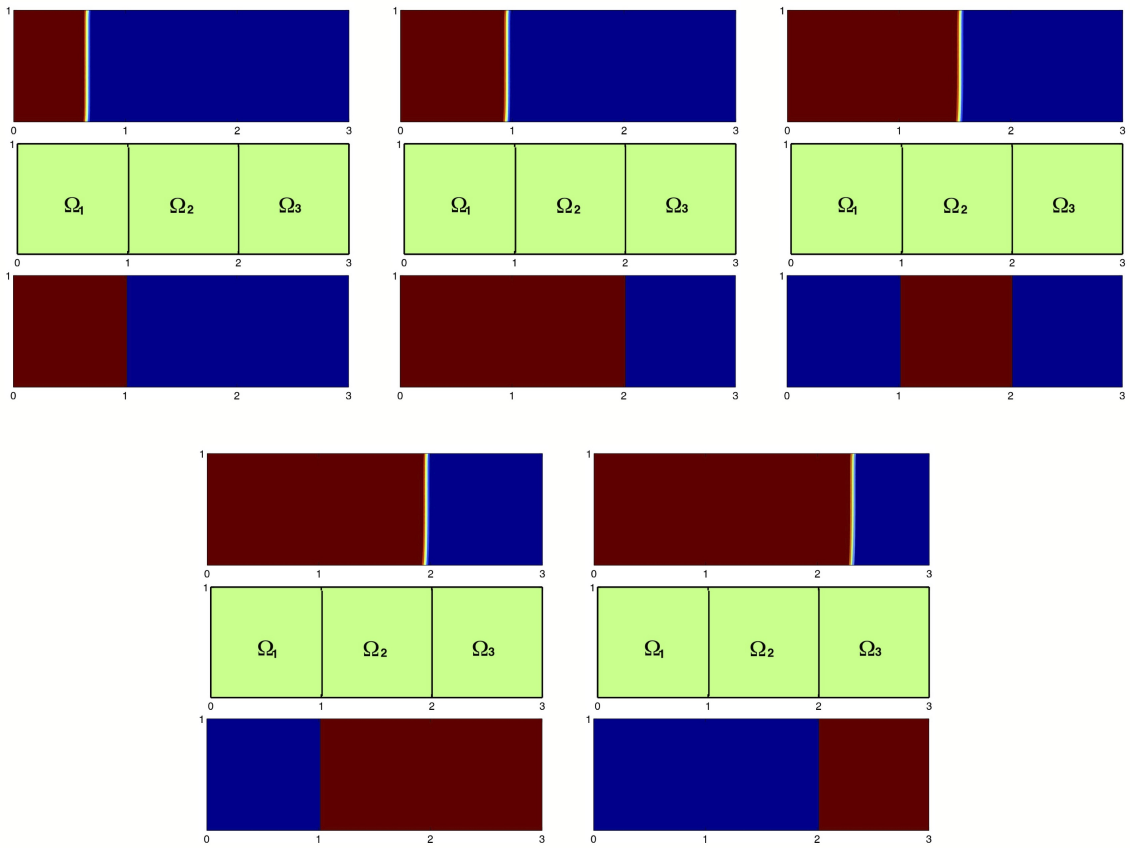

Fig. 2 Propagation of the membrane potential (in red the excited region, top row), the subdomains $\Omega_{1}, \Omega_{2}$, and $\Omega_{3}$ (middle row), and the activated Bidomain region (in red, bottom row).

6. L. Gerardo-Giorda, M. Perego, and A. Veneziani. Optimized Schwarz coupling of Bidomain and Monodomain models in electrocardiology. M2AN, Vol. 45 (2), pp. 309-334, 2011.

7. J. Le Grice, B.H. Smaill, and P.J. Hunter. Laminar structure of the heart: a mathematical model. Am. J. Physiol., 272 (Heart Circ. Physiol.)(41):H2466-H2476, 1995.

8. G.T. Lines, M.L. Buist, P. Grottum, A.J. Pullan, J. Sundnes, and A. Tveito. Mathematical models and numerical methods for the forward problem in cardiac electrophysiology Comput. Visual. Sci,5:215-239, 2003.

9. L. Mirabella, F. Nobile, and A. Veneziani. An a posteriori error estimator for model adaptivity in electrocardiology. Comp. Meth. Appl. Mech. Engrg., (doi:10.1016/j.cma.2010.03.009), 2010 (in press).

10. L. F. Pavarino and S. Scacchi. Multilevel additive Schwarz preconditioners for the Bidomain reaction-diffusion system. SIAM J. Sci. Comp., 31(1):420-443, 2008.

11. M. Pennacchio and V. Simoncini. Algebraic multigrid preconditioners for the bidomain reactiondiffusion system. Appl. Num. Math., 59(12):3033-3050, 2009.

12. M. Potse, B. Dubé, J. Richer, and A. Vinet. A comparison of Monodomain and Bidomain Reaction-Diffusion models for Action Potential Propagation in the Human Heart. IEEE Trans. Biomed. Eng., 53(12):2425-2435, 2006.

13. J. Rogers and A. McCulloch. A collocation-Galerkin finite element model of cardiac action potential propagation. IEEE Transactions on Biomedical Engineering, 41:743-757, 1994.

14. F. B. Sachse. Computational Cardiology. Springer, Berlin, 2004.

15. E.J. Vigmond, R. Weber dos Santos, A.J. Prassl, M. Deo, and G. Plank. Solvers for the caridac bidomain equations. Progress in Biophysics and Molecular Biology, 96(1-3):3-18, 2008.

16. N. Trayanova. Defibrillation of the heart: insights into mechanisms from modelling studies. Experimental Physiology, 91: 323-337, 2006 